\title{
Video Article \\ Heterotopic Renal Autotransplantation in a Porcine Model: A Step-by-Step Protocol
}

\author{
J. Moritz Kaths ${ }^{1,2}$, Juan Echeverri ${ }^{1,3}$, Nicolas Goldaracena ${ }^{1}$, Kristine S. Louis ${ }^{1}$, Paul Yip ${ }^{4}$, Rohan John ${ }^{4}$, Istvan Mucsi ${ }^{5}$, Anand Ghanekar ${ }^{1}$, \\ Darius Bagli ${ }^{6}$, Markus Selzner ${ }^{1}$, Lisa A. Robinson ${ }^{2}$ \\ ${ }^{1}$ Multi Organ Transplant Program, Department of Surgery, Toronto General Hospital \\ ${ }^{2}$ Division of Nephrology, The Hospital for Sick Children \\ ${ }^{3}$ Programa de Doctorat en Medicina, La Universitat Autónoma de Barcelona \\ ${ }^{4}$ Laboratory Medicine and Pathobiology, Toronto General Hospital \\ ${ }^{5}$ Department of Medicine, Toronto General Hospital \\ ${ }^{6}$ Departments of Surgery (Urology) \& Physiology, Developmental \& Stem Cell Biology, The Hospital for Sick Children
}

Correspondence to: J. Moritz Kaths at moritz.kaths@gmail.com

URL: https://www.jove.com/video/53765

DOI: doi:10.3791/53765

Keywords: Medicine, Issue 108, Renal, kidney, organ transplantation, porcine, pig, swine model, auto-allotransplantation, organ shortage, organ preservation technique, marginal graft, delayed graft function (DGF), primary non-function (PNF)

Date Published: 2/21/2016

Citation: Kaths, J.M., Echeverri, J., Goldaracena, N., Louis, K.S., Yip, P., John, R., Mucsi, I., Ghanekar, A., Bagli, D., Selzner, M., Robinson, L.A. Heterotopic Renal Autotransplantation in a Porcine Model: A Step-by-Step Protocol. J. Vis. Exp. (108), e53765, doi:10.3791/53765 (2016).

\section{Abstract}

Kidney transplantation is the treatment of choice for patients suffering from end-stage renal disease. It offers better life expectancy and higher quality of life when compared to dialysis. Although the last few decades have seen major improvements in patient outcomes following kidney transplantation, the increasing shortage of available organs represents a severe problem worldwide. To expand the donor pool, marginal kidney grafts recovered from extended criteria donors (ECD) or donated after circulatory death (DCD) are now accepted for transplantation. To further improve the postoperative outcome of these marginal grafts, research must focus on new therapeutic approaches such as alternative preservation techniques, immunomodulation, gene transfer, and stem cell administration.

Experimental studies in animal models are the final step before newly developed techniques can be translated into clinical practice. Porcine kidney transplantation is an excellent model of human transplantation and allows investigation of novel approaches. The major advantage of the porcine model is its anatomical and physiological similarity to the human body, which facilitates the rapid translation of new findings to clinical trials. This article offers a surgical step-by-step protocol for an autotransplantation model and highlights key factors to ensure experimental success. Adequate pre- and postoperative housing, attentive anesthesia, and consistent surgical techniques result in favorable postoperative outcomes. Resection of the contralateral native kidney provides the opportunity to assess post-transplant graft function. The placement of venous and urinary catheters and the use of metabolic cages allow further detailed evaluation. For long-term follow-up studies and investigation of alternative graft preservation techniques, autotransplantation models are superior to allotransplantation models, as they avoid the confounding bias posed by rejection and immunosuppressive medication.

\section{Video Link}

The video component of this article can be found at https://www.jove.com/video/53765/

\section{Introduction}

Kidney transplantation is the treatment of choice for patients with end-stage renal disease, due to associated lower rates of morbidity and mortality when compared to dialysis ${ }^{1-3}$. Despite major improvements in patient outcomes following kidney transplantation, graft shortage still poses a severe challenge worldwide. The number of patients waiting for a kidney transplant by far exceeds the number of organs available ${ }^{4-6}$. To increase the number of kidneys available for transplantation and to reduce patient waiting times, further sources of kidney grafts are needed.

Commonly, standard criteria donor (SCD) and extended criteria donor (ECD) kidney grafts from donation after brain death (DBD) as well as kidneys recovered from live donors (LDKT) are utilized. Since the 1990s, an increasing number of kidney grafts have been recovered in a donation after circulatory death (DCD) scenario, to further expand the donor pool ${ }^{7,8}$. However, DCD and ECD kidney grafts demonstrate acceptable but decreased outcomes after transplantation, depending on different factors, such as donor age, warm and cold ischemia times, and the preservation technique used ${ }^{9-11}$. Thus, additional research is required to improve the outcome of patients receiving marginal kidney grafts and to further increase the donor pool.

The porcine model of renal transplantation is well established and provides a clinical important scenario to investigate innovative approaches for the improvement of marginal kidney graft outcomes. In contrast to rodent and canine kidneys, which are unilobular, porcine and human 
kidneys are multilobular and are anatomically similar, particularly in regard to the arterial, venous, and urinary collecting systems ${ }^{12,13}$. In addition, porcine and human kidneys demonstrate similarities in the pathophysiology of ischemia reperfusion injury (IRI), biochemistry, and immunological parameters ${ }^{14}$. Thus, porcine renal transplantation is well-suited to investigate new organ preservation methods for marginal kidney grafts ${ }^{15-17}$, model human IRI ${ }^{18}$, study immunological pathways and allograft tolerance ${ }^{19}$, provide surgical training ${ }_{24-22}^{20}$, test new pharmacological therapies ${ }_{23}$, implement new medical devices, and study new immunological mechanisms in xenotransplantation ${ }^{24-26}$ '.

The renal porcine and human transplantation settings are not completely analogous. This article focuses on important technical details that will facilitate successful establishment of a renal autotransplantation model. Species-adapted pre- and postoperative housing, administration of anesthesia with close monitoring, and matched surgical techniques are described in the protocol and demonstrated in the video. Resection of the contralateral native kidney provides the opportunity to assess the function of the transplanted kidney. The placement of venous and urinary catheters and the use of metabolic cages allow more in-depth assessment. For studies aimed at investigating alternative graft preservation methods and mechanisms of IRI, autotransplantation models are superior to allotransplantation models, as they avoid the complications and confounding bias associated with rejection and use of immunosuppressive medications.

\section{Protocol}

All animals received humane care and all studies we carried out in accordance with policies and guidelines of the Canadian Council on Animal Care. All procedures were carried out under Animal Use Protocols that were approved by the University Health Network Institutional Animal Care Committee.

Note: A schematic overview of the study protocol is presented in Figure 1.

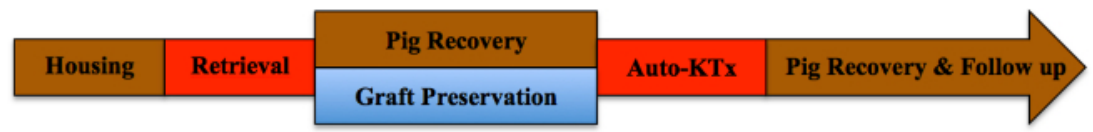

Figure 1. Study protocol. Please click here to view a larger version of this figure.

\section{Animals}

1. Use male Yorkshire pigs $(30 \mathrm{~kg})$ in this protocol.

\section{Kidney Graft Retrieval}

1. Preoperative Procedure

1. House the male Yorkshire pigs in a research facility for at least one week to acclimatize them. Use intramuscular injection of a thirdgeneration cephalosporin, such as ceftiofur, for 3 days to reduce the potential risk of infections with Streptococcus suis and Salmonella. Fast the pigs for a minimum of $6 \mathrm{hr}$ before induction of anesthesia to prevent aspiration.

2. Initiate anesthetization of the pig by an intramuscular injection of ketamine (20 mg/kg), atropine $(0.04 \mathrm{mg} / \mathrm{kg})$, and midazolam (0.3 mg/ $\mathrm{kg})$. Subsequently, transport the animal from the housing facility to the operating room (OR).

3. Place the pig in a supine position on the OR table. Allow the pig to breathe $2 \mathrm{~L}$ of oxygen with $5 \%$ of isoflurane spontaneously. Expose the vocal cords with a laryngoscope and spray them with $2 \%$ lidocaine topical solution to prevent intubation-induced laryngospasm. After intubation with a $6.5 \mathrm{~mm}$ tube, block the cuff with $3-5 \mathrm{ml}$ of air. Note: Capnometry confirms the correct position of the tracheal tube.

4. Decrease the isoflurane gas to $2.5 \%$. Set the ventilator to $14-16$ breaths $/ \mathrm{min}$ and the tidal volume to $10-15 \mathrm{ml} / \mathrm{kg}$ body weight. Monitor the pig closely. Heart rate and oxygen saturation are recorded by pulse oxymetry. Confirm proper anesthetization by reduced heart rate (below 150 beats $/ \mathrm{min}$ ) and blood pressure (below systolic values of $100 \mathrm{mmHg}$ ) as well as absence of porcine movements (no usage of muscle relaxants).

5. Under sterile conditions, introduce an 9.5 Fr. single lumen permanent catheter into the internal jugular vein using Seldinger technique ${ }^{27}$. Briefly, use a needle to puncture the vein. After introducing the guide-wire, replace the needle with the peel-away introducer, followed by replacement of the wire with the vascular catheter. Fix the catheter to the skin using a 3-0 silk or non-absorbable monofilament suture.

1. Administer $500 \mathrm{mg}$ of metronidazole, $1 \mathrm{~g}$ of cefazolin, and $20 \mathrm{mg}$ of pantoprazole. Administer $200 \mathrm{ml}$ of lactated Ringer's solution with $5 \%$ dextrose (D5W) and $1 \mathrm{ml}$ of fentanyl citrate per hour intravenously throughout the surgery. Apply veterinary ophthalmic ointment on eyes to prevent dryness while under anesthesia.

\section{Surgical Procedure}

1. Following sterile disinfection and coverage of the surgical field, perform a midline incision of $25 \mathrm{~cm}$ in length. Insert a retractor. Cover large and small bowels with a towel and position them to the left side for optimal access to the right kidney.

2. Free the ureter and the right kidney itself from any adherent tissue using the cautery.

3. Dissect the right renal vein and artery using the cautery until their origin from the inferior vena cava and the aorta, respectively, are free. To avoid arterial vasospasm, administration of 30-65 mg of papaverine should be considered.

4. After complete renal dissection, tie (silk, 3-0) and cut the ureter distally. Prepare a bowl of ice and a sterile organ bag.

5. First, clamp the renal artery close to the aorta and second, clamp the renal vein close to the vena cava using vessel clamps. Next, resect the kidney graft and immediately cannulate the renal artery with a renal artery cannula. Use $500 \mathrm{ml}$ of ice-cold histidinetryptophan-ketoglutarate (HTK) solution to flush out the blood. Store the kidney on ice until transplantation.

6. In situ, close the remaining renal artery with a ligature (silk, 2-0) and the renal vein with a running suture (prolene, 6-0). 
7. After checking the dissected area for bleeding, close the abdominal wall with a running suture (monofil, 1) and the skin with a 3-0 silk or non-absorbable monofilament suture..

3. Postoperative Procedure

1. Fix the venous catheter subcutaneously with a suture (silk, 3-0) and tunnel it to the pig's back to prevent unwanted manipulation. After placing the pig prone, suture (silk, 3-0) the catheter firmly to the skin.

2. Wean the pig from the ventilator and let it recover in its housing area after extubation. Administer Ringer's lactate intravenously for volume expansion and administer $0.3 \mathrm{mg}$ buprenorphine for analgesia. Do not leave an animal unattended until it has regained sufficient consciousness to maintain sternal recumbency.

\section{Kidney Graft Transplantation}

1. Preoperative Procedure

1. Anesthetize the pig using intravenous injection of propofol (1-2 $\mathrm{mg} / \mathrm{kg}$ body weight) followed by a continuous infusion of propofol at a rate of 50-100 mg/hr. Re-intubate the pig as described in step 2.1.3 and 2.1.4 and set the isoflurane gas to 3-4\%.

2. Administer $1 \mathrm{~g}$ of cefazolin and $20 \mathrm{mg}$ of pantoprazole i.v. During surgery, use the same anesthetic protocol as described in 2.1 .4 .

3. Following sterile disinfection, make a cut of $4 \mathrm{~cm}$ next to the trachea. Dissect the tissue to expose the carotid artery. Pass over-holt forceps and a silk tie (2-0) around the artery. Use the Seldinger technique to introduce a plastic catheter to continuously measure the arterial pressure throughout the surgery. Alternatively, non-invasive blood pressure measurement techniques can be utilized.

2. Surgical Procedure

1. After sterile disinfection, reopen the abdominal cavity by cutting the stitches of the skin and fascia sutures, reintroduce the surgical retractor to expose the abdominal cavity, and reposition the bowel to the left side to allow better access to the infrarenal vessels.

2. Transplant the preserved kidney graft end-to-side to the infrarenal vena cava and aorta. Therefore, dissect vena cava and aorta over $5-8 \mathrm{~cm}$ above the iliac bifurcation using pickups and cautery. If possible, do not disturb the lymphatic vessels; if not possible, close them with 5-0 prolene sutures.

3. After completing the dissection, check for bleeding and remove remaining tissue from the vessels. Ensure that complete clamping of the vena cava and aorta with a Satinsky clamp is feasible.

4. Next, resect the contralateral (left) kidney. To do so, position the bowel to the right; dissect the ureter, the kidney itself, the renal vein, and the renal artery from adherent tissue. Tie the ureter and blood vessels and resect the kidney. Check for bleeding.

5. Reposition the bowel to the left to expose the infrarenal aorta and vena cava. Inject heparin (100 IU/ kg body weight) and wait for at least 2 min.

6. Venous Anastomosis:

1. Use a Satinsky clamp to completely clamp the vena cava and make a slit incision that matches the size of the opening of the renal vein, using an 11 blade. Pott scissors can be used to further extend the slit.

2. After wrapping the kidney into a cloth containing sterile ice, remove it from the ice and position it into the surgical field. Use two double-armed 6-0 prolene sutures to perform a cranial and a caudal corner stitch.

3. Approximate the kidney, tie the upper corner and perform a running suture using 6-0 prolene, starting with the back wall. After having finished $2 / 3$, use the other end of the tie to complete the suture at the front side. After tying the cranial stitches, tie the stitches at the caudal corner.

4. Position a bulldog clamp on the renal vein and open the Satinsky clamp. Check the anastomosis for bleeding.

7. Arterial Anastomosis:

1. Use the Satinsky clamp again to completely clamp the aorta. Use an 11 blade to make a slit incision, matching the opening of the renal artery. Use a $4.0 \mathrm{~mm}$ round punch to secure a clean opening.

2. Use one 6-0 prolene suture to perform the arterial anastomosis, starting at the recipient side. Ensure that the arterial endothelium is included in each suture to prevent a dissection. Meanwhile, start a continuous drip of $10 \mathrm{ml}$ norepinephrine $(16 \mathrm{mg} / 250 \mathrm{ml})$ diluted in $500 \mathrm{ml}$ of Ringer's lactate and titrate to keep the systolic pressure above $100 \mathrm{mmHg}$.

3. Inject verapamil intra-arterially before completion of the arterial anastomosis and administer papaverine topically to the outside of the vessel to prevent vasospasm.

4. Position a bulldog clamp on the renal artery and open the Satinksy clamp. Check the anastomoses for bleeding

5. Unwrap the kidney from the cloth and remove the ice. Open the venous bulldog clamp first, followed by the arterial bulldog clamp. After reperfusion, urine production should start immediately.

6. Use cloth to secure a favorable position for the transplanted graft and maintain a homogenous reperfusion.

8. Ureteral Anastomosis:

1. Use Pott scissors to open the ureter from the graft and the recipient over a longitudinal length of $0.5 \mathrm{~cm}$.

2. Use two 6.0 polyester, poly ( $\mathrm{p}$-dioxanone) sutures for the side-to-side ureteral anastomosis. Perform a corner stich at each side, then run the back wall in a continuous manner first, followed by the front wall.

3. After checking for bleeding, remove the cloth and wrap some of the small bowel around the kidney to hold it in position. Close the abdominal wall with two monofil 1 sutures. Close the skin with 3-0 silk or non-absorbable monofilament suture.

4. Maintain the systolic pressure above $100 \mathrm{mmHg}$ continuously by carefully titrating the norepinephrine infusion until the pig has been placed into prone position.

3. Postoperative Procedure

1. After abdominal closure as mentioned above, keep the pig warm using a heating pad and heat-circulating blanket. Remove the arterial line, close the puncture hole in the artery with a 6-0 prolene stich and close the incision site. 
2. Turn the pig onto prone position, stop the norepinephrine drip and wean the pig from the ventilator. Allow the pig to recover in its housing area and monitor it closely to ensure its smooth recovery from the procedure. Take blood gas samples each hr via the implanted jugular catheter. Provide Ringer's lactate to substitute volume and administer $0.3 \mathrm{mg}$ buprenorphine for analgesia.

3. Following extubation, monitor the pig closely until it is able to drink spontaneously. Do not leave an animal unattended until it has regained sufficient consciousness to maintain sternal recumbency. Do not return an animal that has undergone surgery to the company of other animals until fully recovered.

\section{Postsurgical Follow Up}

1. Administer $0.3 \mathrm{mg}$ buprenorphine i.v. every $8 \mathrm{hr}$ for at least 2 days post surgery or longer if needed. Routinely administer a single prophylactic dose of antibiotic during surgery. In the event of signs of infection, administer cefazolin $1 \mathrm{~g}$ i.v. twice per day and metronidazole i.v. once per day until clinical improvement occurs. Administer Ringer's lactate until the pig drinks sufficient water. 1,000 IU heparin can be used to lock the catheter to prevent clotting.

2. Collect venous blood samples via the jugular catheter and urinary samples to assess the pig's clinical condition and renal function.

3. For euthanasia, induce anesthesia of the pig with propofol i.v. $(5-10 \mathrm{ml})$ and maintain it with isoflurane $5 \%$. Intubate the pig as described above. After relaparatomy and renal tissue sample collection, induce cardiac arrest by intravenous injection of $40 \mathrm{mval} \mathrm{KCl}$.

\section{Representative Results}

In the following, the results of renal autotransplantation experiments $(n=4)$ are demonstrated. After the initial graft retrieval, the pigs recovered in their housing area. Meanwhile, the kidney grafts were stored on ice for a mean time of $7 \mathrm{hr} 35 \mathrm{~min}$ ( $\pm 18 \mathrm{~min})$. After reinduction of anesthesia and repeat laparotomy, the contralateral kidneys were resected and the cold-stored grafts transplanted heterotopically as described. After weaning from the ventilator, pigs were recovered from surgery and followed up for 10 days (see Figure 1). Daily (1-4 post-operative day; pod) or every second day (6-10 pod) blood samples were collected to perform blood gas analyses; to assess the renal function, serum creatinine and blood urea nitrogen (BUN) values were estimated. For comparison, the results of one allotransplanted kidney graft are presented. For immunosuppression, this pig received cyclosporine $100 \mathrm{mg}$ p.o. and cortisone $250 \mathrm{mg} \mathrm{i.v.} \mathrm{b.i.d.} \mathrm{The} \mathrm{surgical} \mathrm{technique} \mathrm{used} \mathrm{was} \mathrm{the} \mathrm{same} \mathrm{as} \mathrm{in}$ the autotransplant protocol; no warm ischemia time was applied.

All pigs were in good clinical condition during the follow up period. The serum creatinine and BUN values revealed the highest increase at day one after surgery (Crea $2.8 \pm 0.7 \mathrm{mg} / \mathrm{dl}$, BUN $25.3 \pm 7 \mathrm{mg} / \mathrm{dl}$ ) and decreased until pod 10 (Crea $1.7 \pm 0.4 \mathrm{mg} / \mathrm{dl}$, BUN $10.7 \pm 4 \mathrm{mg} / \mathrm{dl}$ ) close to the initial baseline values. The allotransplanted kidney graft demonstrated higher creatinine and BUN values after good initial graft function, when compared to the autografts, most likely due to rejection (Figure 2 and 3). Acid-base hemostasis (Figure 4) and electrolyte levels (Figure 5) were stable without intervention. Histological examination showed preserved tubulointerstitium in the autotransplanted kidney (Figure 6), and diffuse interstitial inflammation, tubulitis, and glomerulitis in the allotransplanted kidney (Figure 7).

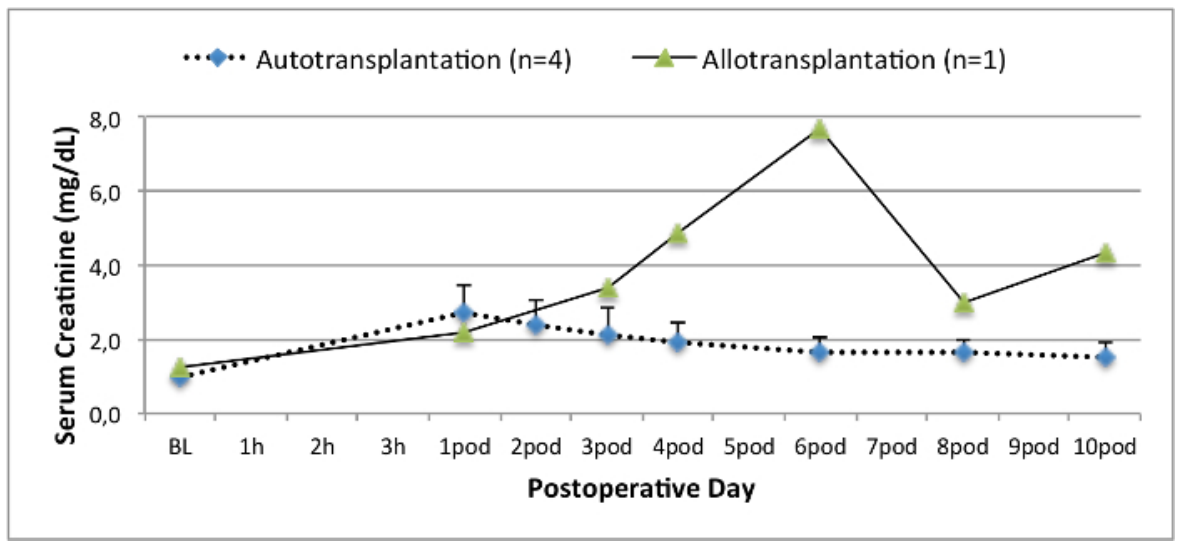

Figure 2. Serum creatinine values. Serum creatinine values (mean and standard deviation) for baseline and 10 after surgery. Please click here to view a larger version of this figure. 


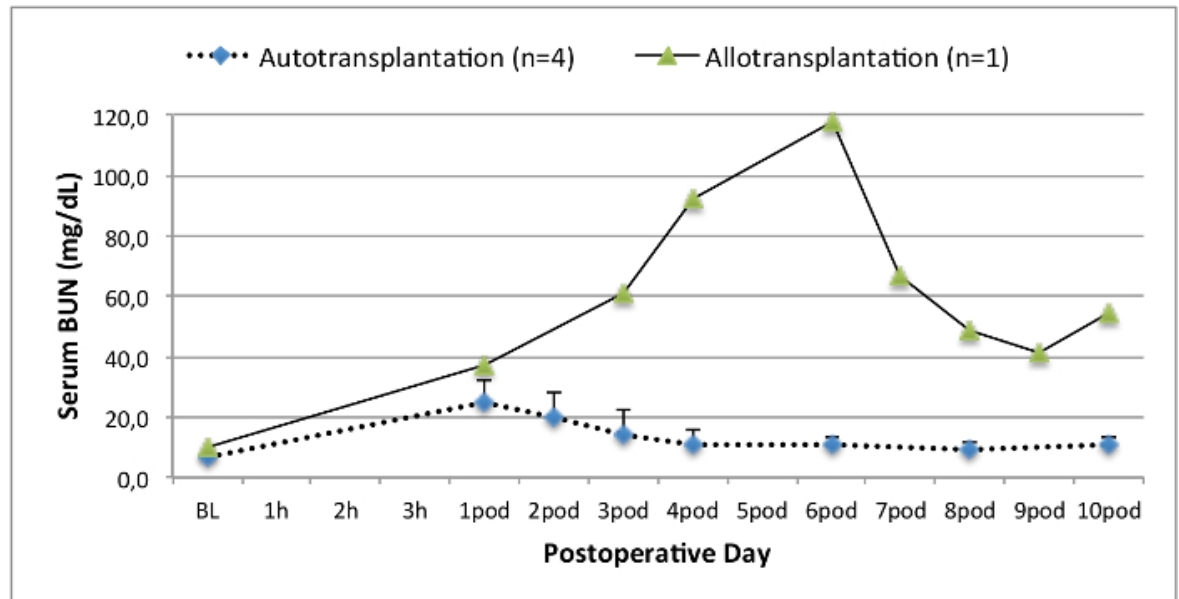

Figure 3. Serum BUN values. Serum BUN values (mean and standard deviation) for baseline and 10 days after surgery. Please click here to view a larger version of this figure.

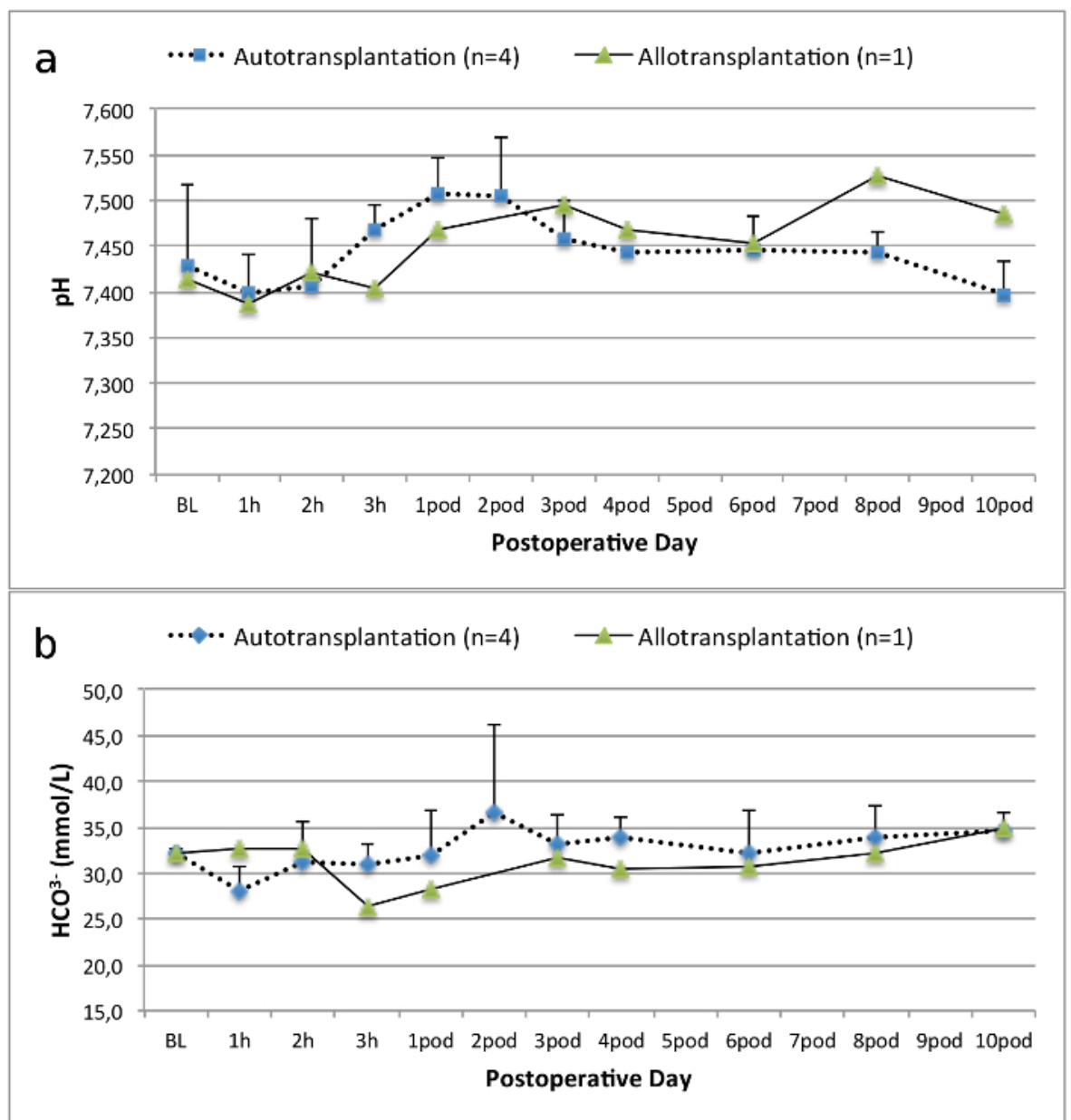

Figure 4. Acid-base hemostasis. Acid-base hemostasis (mean and standard deviation) for baseline and 10 days after surgery. Please click here to view a larger version of this figure. 


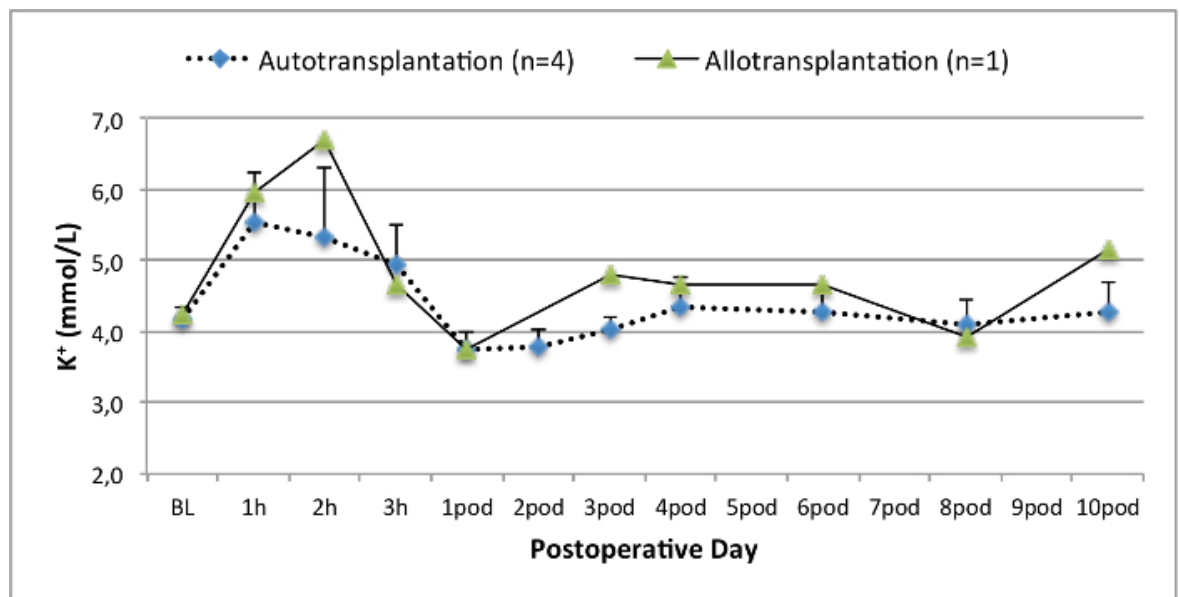

Figure 5. Electrolyte levels. Electrolyte levels (mean and standard deviation) for baseline and 10 days after surgery. Please click here to view a larger version of this figure.

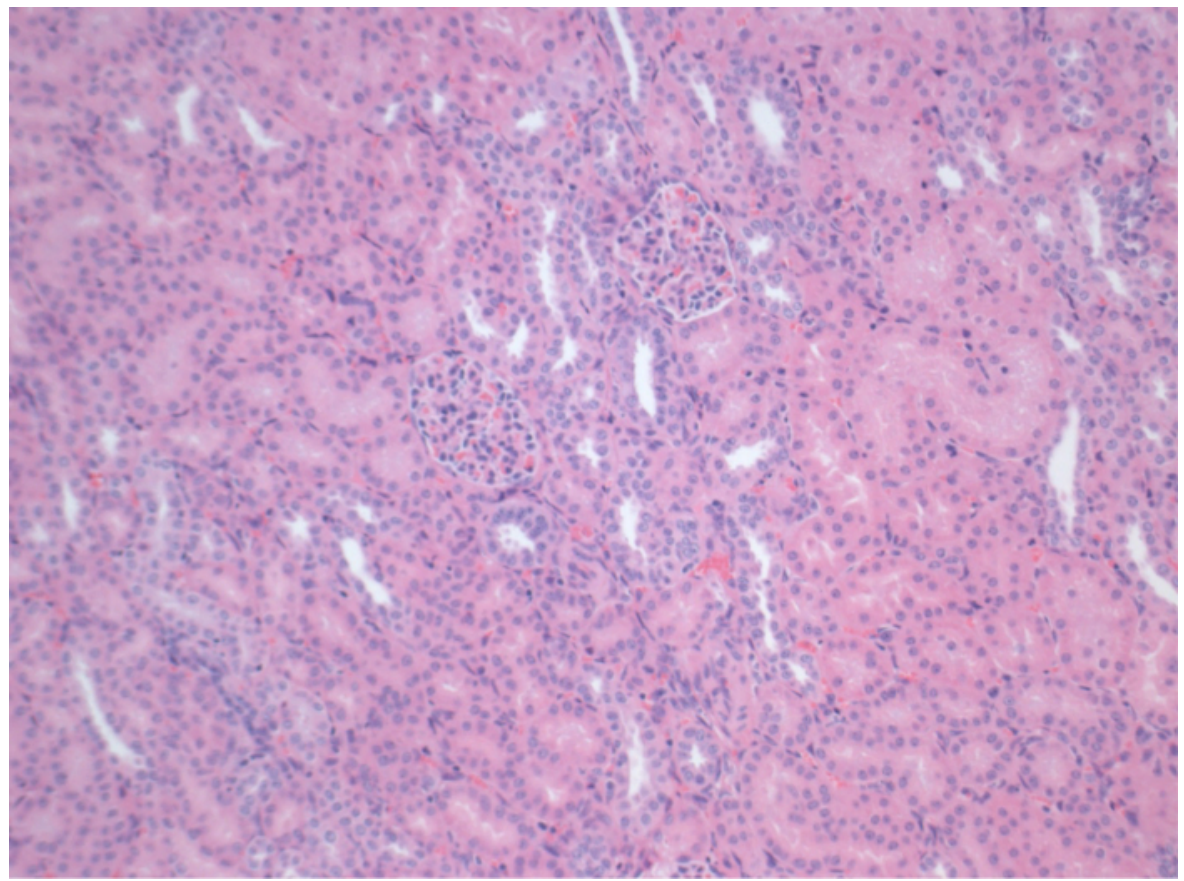

Figure 6. Histology (H\&E), 100X magnification. Normal tubulointerstitium in the autotransplanted kidney 10 days after surgery. Please click here to view a larger version of this figure. 


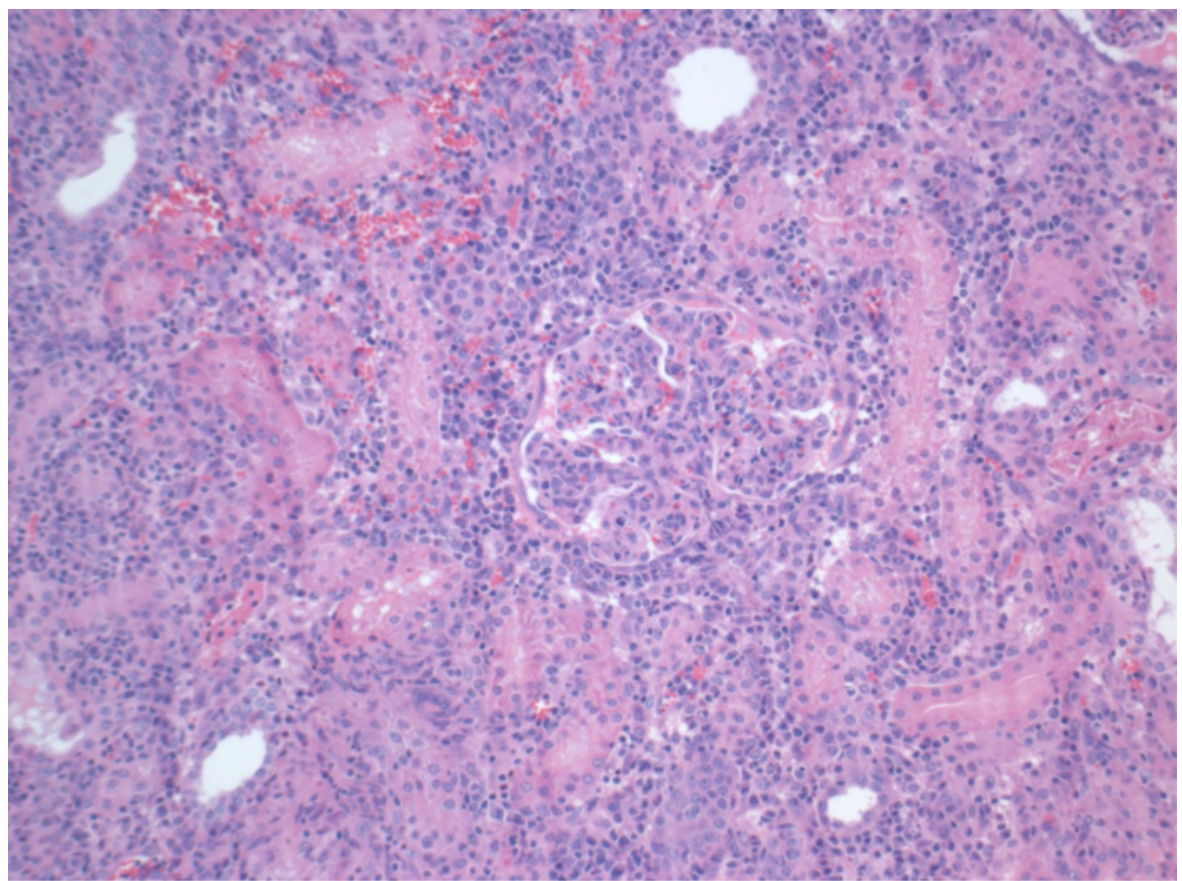

Figure 7. Histology (H\&E), 100X magnification. Extensive interstitial inflammation, tubulitis, and glomerulitis, consistent with rejection, in the allotransplanted kidney 10 days after surgery. Please click here to view a larger version of this figure.

\section{Discussion}

The model of porcine kidney transplantation provides a unique opportunity to further the field of human transplantation due to similarities in surgical aspects, physiology, biochemistry, and immunology ${ }^{14}$.

Depending on the purpose of the experimental study, the model of renal autotransplantation has several advantages compared to the allotransplantation model. Although several groups report good renal graft function after allotransplantation ${ }^{28}$, immunosuppression in pigs is challenging, especially in renal transplantation. Preoperative blood sample analyses to ensure compatibility for swine leukocyte antigen (SLA) are feasible, but expensive and impractical ${ }^{14}$. Postoperatively, proposed immunosuppressive agents such as tacrolimus and cyclosporine (calcineurin inhibitors, $\mathrm{CNI}$ ) are administered orally or i.v. ${ }^{28}$. Oral administration is impractical, as pigs usually refuse to swallow oral medication. Furthermore, intestinal obstructions might obviate sufficient absorption of immunosuppressive medications and maintenance of therapeutic drug levels. The continuous infusion of CNI's i.v. in active animals is technically demanding. I.v. bolus administration leads to high peak values, which cause toxicity. Thus, for the investigation of new preservation techniques, the model of renal autotransplantation has several advantages. In the representative results of the allotransplantated kidney graft demonstrated above, a delayed and increased peak of creatinine and BUN indicate rejection, which was demonstrated by histological assessment.

The porcine model of autotransplantation has previously been used to investigate new preservation techniques ${ }^{14,18,29}$. However, the reported postoperative serum creatinine and BUN values of autotransplanted pigs in a heart-beating scenario vary considerably depending on the experimental system ${ }^{22,30}$. The heart-beating donor protocol we present here results in a low postoperative serum creatinine peak of $2.8 \mathrm{mg} / \mathrm{dl}$ $( \pm 0.7)$ and BUN peak of $25.3 \mathrm{mg} / \mathrm{dl}( \pm 7.4)$. These results are comparable with the low peak values presented by Hanto and colleagues ${ }^{28}$ and Snoeijs and colleagues ${ }^{31}$.

To ensure a successful outcome after renal transplantation in a porcine autotransplantation model, we have identified several key technical factors that minimize the rate of certain complications. The use of histidine-tryptophan-ketoglutarate solution (HTK) reduces the risk of vasospasm due to its lower content of potassium when compared to University of Wisconsin (UW) solution. To further decrease the risk of vasospasm at the point of reperfusion, verapamil can be injected into the renal artery, and papaverine can be administered topically during retrieval and after reperfusion. In addition, a continuous drip of norepinephrine titrated to maintain the systolic blood pressure above $100 \mathrm{mmHg}$ ensures a homogeneous reperfusion. It is useful to maintain this blood pressure at least until the pig is positioned prone. Furthermore, the positioning of the transplanted graft is important to prevent kinking of the newly anastomosed blood vessels. Therefore, it is helpful to resect the contralateral left kidney prior to sewing the anastomoses of the graft to avoid extensive mechanical manipulation. After finishing the ureteral anastomosis, wrapping small intestine around the transplanted graft secures its position after closure of the abdominal wall. Complications such as bowel obstructions due to kinking of the intestine are rarely observed but can lead to severe complications, including ileus, bowel perforation, and death. Overall, accurate surgical technique, attentive anesthesia and close monitoring during follow up ensure good clinical outcome and graft function.

Arterial and venous anastomoses can be performed using different techniques. Orthotopic placement of the graft allows end-to-end anastomoses of the renal artery and vein. In the case of heterotopic transplantation, the graft can be positioned in the contralateral renal fossa for end-to-end anastomoses, onto the iliac vessels, or the distal aorta directly. Heterotopic transplantation with anastomoses to aorta and cava directly in end-toside technique are preferred in this model as it can reduce the risk of thrombosis and vasospasm ${ }^{32}$. Anatomical variations with very early venous bifurcations might lead to the need of sewing two separate venous anastomoses. If the artery or vein are relatively short, the graft can be turned 
$180^{\circ}$ to gain length of the vessels. Ureteral side-to-side anastomosis can achieve good experimental results without complicating strictures or urinary leak.

In general, the porcine model of renal transplantation offers advantages compared to other animal models. As described above, certain similarities exist between the porcine and the human setting, which allows relatively fast translation of new techniques into clinical practice. The technique of transplantation is technically easier compared to rodent models. In addition, by placement of venous catheters, peripheral blood samples can be collected easily and processed for further investigation. The collection of urine allows further assessment of kidney injury and function. To collect urine samples, a percutaneous catheter can be inserted into the urinary bladder. To avoid manipulation by the pig, the distal end should be tunneled subcutaneously to the back of the animal. Another option for urine collection is the use of metabolic cages, which allow prolonged collection periods to estimate the creatinine clearance and concentration of additional biomarkers in the urine. Sonography, CT scans, and MRI images are possible. Donation after circulatory death protocols can be mimicked by applying warm ischemia prior to retrieval. Furthermore, pigs are relatively easy to handle if castrated to limit their aggressive behavior.

Disadvantages include the high costs of animal purchase, housing, surgical and other medical equipment, and manpower. These factors mean that it is not feasible to include large numbers of animals in each study group. Furthermore, compared to rodent models, a limited number of references are available in the literature for pig normative biological data. As an alternative for the assessment of new developed techniques, such as novel preservation methods, other groups have described the normothermic ex vivo reperfusion as an alternative to renal transplantation 33,34 . This technique is easier to perform and less expensive. However, standardized kidney graft transplantation provides a model more similar to the clinical practice and allows longer follow up periods. Therefore, it serves for a more realistic graft assessment.

In conclusion, the porcine model of heterotopic renal autotransplantation provides a clinical important scenario to investigate innovative novel approaches for the improvement of kidney graft outcomes. In particular, this protocol features important technical details that will facilitate successful establishment of a renal autotransplantation model and allows the rapid translation of new findings to clinical trials.

\section{Disclosures}

The authors have nothing to disclose.

\section{Acknowledgements}

We thank the Sorin Group (Milano, Italy), XVIVO Perfusion Inc. (Goteborg, Sweden), and Braun AG (Melsungen, Germany) for their support. We highly appreciate the support of the John David and Signy Eaton Foundation.

\section{References}

1. Wolfe, R. A., Ashby, V. B., et al. Comparison of mortality in all patients on dialysis, patients on dialysis awaiting transplantation, and recipients of a first cadaveric transplant. N Engl J Med. 341 (23), 1725-1730 (1999).

2. Ingsathit, A., Kamanamool, N., Thakkinstian, A., \& Sumethkul, V. Survival advantage of kidney transplantation over dialysis in patients with hepatitis C: a systematic review and meta-analysis. Transplantation. 95 (7), 943-948 (2013).

3. Tonelli, M., Wiebe, N., et al. Systematic review: kidney transplantation compared with dialysis in clinically relevant outcomes. Am $J$ Transplant. 11 (10), 2093-2109 (2011).

4. Global Observatory on Donation \& Transplantation. Source: http://www.transplant-observatory.org/Pages/home.aspx (2014).

5. Matas, A.J. et al. OPTN/SRTR Annual Data Report 2012: Kidney. Am J Transplant. 14 Suppl 1. (2014).

6. Rahmel, A. Annual Report 2013 - Eurotransplant International Foundation. Source: https://www.eurotransplant.org/cms/mediaobject.php? file=AR20135.pdf (2013).

7. Morrissey, P. E., \& Monaco, A. P. Donation after circulatory death: current practices, ongoing challenges, and potential improvements. Transplantation. 97 (3), 258-264 (2014).

8. Maggiore, U., Oberbauer, R., et al. Strategies to increase the donor pool and access to kidney transplantation: an international perspective. Nephrol Dial Transplant. 30 (2), 217-22. (2014).

9. Summers, D. M., Johnson, R. J., Hudson, A., Collett, D., Watson, C. J., \& Bradley, J. A. Effect of donor age and cold storage time on outcome in recipients of kidneys donated after circulatory death in the UK: a cohort study. Lancet. 381 (9868), 727-734 (2013).

10. Wadei, H. M., Heckman, M. G., et al. Comparison of kidney function between donation after cardiac death and donation after brain death kidney transplantation. Transplantation. 96 (3), 274-281 (2013).

11. Moers, C., Smits, J. M., et al. Machine perfusion or cold storage in deceased-donor kidney transplantation. N Engl J Med. 360 (1), 7-19 (2009).

12. Pereira-Sampaio, M. A., Favorito, L. A., \& Sampaio, F. J. B. Pig kidney: anatomical relationships between the intrarenal arteries and the kidney collecting system. Applied study for urological research and surgical training. J Urol. 172 (5 Pt 1), 2077-2081 (2004).

13. Bagetti Filho, H. J. S., Pereira-Sampaio, M. A., Favorito, L. A., \& Sampaio, F. J. B. Pig kidney: anatomical relationships between the renal venous arrangement and the kidney collecting system. J Urol. 179 (4), 1627-1630 (2008).

14. Giraud, S., Favreau, F., Chatauret, N., Thuillier, R., Maiga, S., \& Hauet, T. Contribution of large pig for renal ischemia-reperfusion and transplantation studies: the preclinical model. J Biomed Biotechnol. 2011 (21), 532127 (2011).

15. Gallinat, A., Paul, A., et al. Role of oxygenation in hypothermic machine perfusion of kidneys from heart beating donors. Transplantation. 94 (8), 809-813 (2012).

16. Thuillier, R., Allain, G., et al. Benefits of active oxygenation during hypothermic machine perfusion of kidneys in a preclinical model of deceased after cardiac death donors. J Surg Res. 184 (2), 1174-1181 (2013). 
17. Hosgood, S. A., Barlow, A. D., Yates, P. J., Snoeijs, M. G. J., van Heurn, E. L. W., \& Nicholson, M. L. A pilot study assessing the feasibility of a short period of normothermic preservation in an experimental model of non heart beating donor kidneys. J Surg Res. 171 (1), $283-290$ (2011).

18. Delpech, P. O., Thuillier, R., et al. Effects of warm ischaemia combined with cold preservation on the hypoxia-inducible factor $1 a$ pathway in an experimental renal autotransplantation model. Br J Surg. 101(13),1739-50 (2014).

19. Kirk, A. D. Crossing the bridge: large animal models in translational transplantation research. Immunol Rev. 196, 176-196 (2003).

20. Golriz, M., Hafezi, M., et al. Do we need animal hands-on courses for transplantation surgery? Clin Transplant. 27 Suppl 25 (s25), 6-15 (2013).

21. He, B., Musk, G. C., Mou, L., Waneck, G. L., \& Delriviere, L. Laparoscopic surgery for kidney orthotopic transplant in the pig model. JSLS. 17 (1), 126-131 (2013).

22. Faure, A., Maurin, C., et al. An experimental porcine model of heterotopic renal autotransplantation. Transplant Proc. 45 (2), $672-676$ (2013).

23. Hosgood, S. A., Yates, P. J., \& Nicholson, M. L. 1400W reduces ischemia reperfusion injury in an ex-vivo porcine model of the donation after circulatory death kidney donor. World J Transplant. 4 (4), 299-305 (2014).

24. Ghanekar, A., Mendicino, M., et al. Endothelial induction of fgl2 contributes to thrombosis during acute vascular xenograft rejection. $J$ Immunol. 172 (9), 5693-5701 (2004).

25. Ghanekar, A., Lajoie, G., et al. Improvement in rejection of human decay accelerating factor transgenic pig-to-primate renal xenografts with administration of rabbit antithymocyte serum. Transplantation. 74 (1), 28-35 (2002).

26. Cowan, P. J., Cooper, D. K. C., \& d'Apice, A. J. F. Kidney xenotransplantation. Kidney Int. 85 (2), 265-275 (2014).

27. Seldinger, S. I. Catheter replacement of the needle in percutaneous arteriography; a new technique. Acta radiol. 39 (5), $368-376$ (1953).

28. Hanto, D. W., Maki, T., et al. Intraoperative administration of inhaled carbon monoxide reduces delayed graft function in kidney allografts in Swine. Am J Transplant. 10 (11), 2421-2430 (2010).

29. Maathuis, M.-H. J., Manekeller, S., et al. Improved kidney graft function after preservation using a novel hypothermic machine perfusion device. Ann Surg. 246 (6), 982-91 (2007).

30. Gallinat, A., Paul, A., et al. Hypothermic reconditioning of porcine kidney grafts by short-term preimplantation machine perfusion. Transplantation. 93 (8), 787-793 (2012).

31. Snoeijs, M. G., Matthijsen, R. A., et al. Autologous transplantation of ischemically injured kidneys in pigs. J Surg Res. 171 (2), $844-850$ (2011).

32. Golriz, M., Fonouni, H., Nickkholgh, A., Hafezi, M., Garoussi, C., \& Mehrabi, A. Pig kidney transplantation: an up-to-date guideline. Eur Surg Res. 49 (3-4), 121-129 (2012).

33. Hosgood, S. A., Bagul, A., Yang, B., \& Nicholson, M. L. The relative effects of warm and cold ischemic injury in an experimental model of nonheartbeating donor kidneys. Transplantation. 85 (1), 88-92 (2008).

34. Hoyer, D. P., Gallinat, A., et al. Influence of oxygen concentration during hypothermic machine perfusion on porcine kidneys from donation after circulatory death. Transplantation. 98 (9), 944-950 (2014). 